\title{
PROCÉDÉS D'AMÉLIORATION DES QUALITÉS DE RÉGLAGE DES GROUPES HYDROELECTRIQUES
}

\section{PROCESSES FOR THE IMPROVEMENT OF THE REGULATION CHARACTERISTICS OF HYDRO-ELECTRIC UNITS}

par M. P. ALMERAS,

Ingénieur aux Ateliers NEYRPIC,

Professeur à l'Institut Polytechnique de Grenoble.

English Synopsis p. 6

On avait depuis longtemps remarqué que les groupes hydroélectriques avaient des possibilités limitées en ce qui concerne le réglage de la fréquence. Lorsqu'on voulait leur donner de bonnes qualités de réglage (grande rapidité de réponse), on faisait apparaître du même coup des phénomènes d'instabilité. Pour faire disparaître cette instabilité, on était obligé de diminuer la rapidité de réponse. On a voulu mettre en cause la conception même des régulateurs de turbines hydrauliques ou leur construction.

On sait, maintenant, que les qualités de réglage des groupes hydroélectriques sont affectées principalement par les phénomènes d'inertie de leur fluide moteur, l'eau. C'est ainsi que, par exemple, dans le cas des basses et moyennes chutes, et en l'absence de phénomènes d'autoréglage (en particulier dans le cas où le réseau alimenté est purement résistant, maintenu à tension constante) et pour un choix optimum des caractéristiques des régulateurs, la stabilité de marche réclame la condition:

$$
\frac{1}{K_{0}}=\tau^{\prime}>3.74 \frac{\rho}{z}
$$

où :

- $\tau^{\prime}$ est la lenteur de réglage (temps que met le groupe à faire varier sa puissance de $1 \%$ sous un écart de fréquence de $1 \%$ ). Son inverse $K_{0}$ est la "promptitude de réglage» ou "rapidité de réponse $»$.

- : est le temps de lancer ou inertie spécifique, qui caractérise l'inertie des pièces tournantes du groupe $=\frac{I_{(1)}{ }^{2}}{W_{p}}$
- 5 est le temps, caractéristique de l'inertie hydraulique du fluide moteur: $\theta=\frac{\Sigma L V}{g H} \quad$ C'est aussi en quelque sorte, un temps de lancer: c'est le temps que mettrait l'eau de la conduite à atteindre la vitesse de régime sous la chute normale.

La condition ci-dessus exprime donc que plus l'inertie hydraulique $\theta$ sera grande et l'inertie mécanique : sera petite, et plus la lenteur de réglage $\tau^{\prime}$ sera grande, c'est-à-dire plus le régulateur sera lent à régler.

Nous n'insisterons pas sur ces définitions ni sur la démonstration de l'équivalence des systèmes à asservissement temporaire ou à accéléromètre qui ont fait l'objet d'une importante série d'articles parus soit dans la revue "LA HOUILLE BLANCHE » en 1946-1947 et 1948, soit dans le «BULLETIN TECHNIQUE DE LA SUISSE ROMANDE » (M. GADEN), soit dans la "SCHWEIZERISCHE BAUZEITUNG» (M. STEIN).

Rappelons seulement que l'équation différentielle qui régit le comportement d'un groupe est, en général, le résultat de la combinaison de trois équations:

a) La loi de réponse du régulateur, qui s'écrit :

$$
\frac{d}{d t} \Delta y=-\frac{1}{z^{\prime}} \Delta \omega-K_{1} \frac{d}{d t} \Delta \omega
$$

dans le cas du régulateur accélérotachymétrique, et :

$\frac{d}{d t} \Delta y=-\frac{1}{\sigma T_{r}} \Delta\left(,-\frac{1}{\sigma}-\frac{d}{d t} \Delta(n)\right.$ 
dans le cas du régulateur à asservissement temporaire.

Dans ces équations, y est l'ouverture de la turbine, et $\Delta y$ son écart par rapport à l'ouverture de régime $y_{1}$.

$\triangle$ a) est l'écart de la vitesse par rapport à la vitesse de régime ', $y_{0}$, rapporté à cette vitesse de régime :

$$
\Delta,=\frac{\omega-\omega_{1}}{(1)}
$$

La comparaison entre les équations (1) et ( $1^{\prime}$ ) montre que la lenteur de réglage du régulateur à dash-pot est le produit du degré d'asservissement par la rigidité $T$, du dash-pot.

$$
z^{\prime} \sim \triangle T \text {, }
$$

et que le coefficient d'action accélérométrique d'un tel régulateur n'est que l'inverse du degré d'asservissement.

$$
K_{1} \sim \frac{1}{\sigma}
$$

Le dosage accélérotachymétrique a donc pour équivalent dans le régulateur à dash-pot la rigidité $T$.

$$
m=K_{1} \tau^{\prime} \sim T \text {, }
$$

D) L'équation générale de la régulation:

$$
\mathrm{I} \frac{\mathrm{d}(1)}{\mathrm{dt}}=\mathrm{C}_{m}-\mathrm{C}_{\mathrm{r}}
$$

qui exprime que l'accélération $\frac{d \omega}{d t}$ est propor. tionnelle à la différence entre les couples moteur et résistant, et qui s'écrit ici :

$$
=\frac{d}{d t} \Delta\left(n=\Delta y+\frac{3}{2} y_{0} \Delta h\right.
$$

le terme $3 / 2 y_{0} \triangle h$ exprimant seulement que la puissance est proportionnelle à l'exposant $3 / 2$ de la chute

c) L'équation du coup de bélier, qui s'écrit :

$$
\Delta h=-0\left(\frac{d}{d t} \Delta y+\frac{y_{0}}{2} \frac{d}{d t} \Delta h\right)
$$

qui donne la surpression $\Delta h$ rapportée à la chute

$$
\begin{gathered}
\text { normale }\left(\Delta h=\frac{H-H_{0}}{H_{0}}\right) \text { en fonction de la } \\
\text { vitesse de vannage } \frac{d}{d t} \Delta y \text { lorsquion peut }
\end{gathered}
$$

négliger l'élasticité de l'eau (basses et moyennes chutes)

Seule la première équation, celle de la loi de réponse, dépend du régulateur lui-même, les deux autres en sont indépendantes.

Quoiqu'il en soit, la condition de stabilité que l'on déduit de ces équations

$$
\frac{\because}{0}>K=3,74
$$

est génante surtout dans les basses chutes. En effet, " est alors assez grand et peut atteindre 4 ou 5" au lieu de 1" à 1"5 dans les installations moyennes ou hautes. De plus, le temps de lancer diminue de plus en plus par suite des progrès de la technique des constructions électriques, il varie de 5 à 10" actuellement. Ceci conduit à augmenter la lenteur de réglage dans des proportions telles qu'on a eu peur que la fréquence ne puisse plus être réglée de manière satisfaisante.

En fait, le péril n'était pas grave par suite de l'interconnection qui, en diminuant les valeurs des variations relatives de puissance, permettait d'avoir une fréquence bien réglée même avec de grandes lenteurs de réglage. Le péril existait cependant en cas de marche accidentelle sur réseau séparé. II était donc intéressant de diminuer le coefficient 3,74 du deuxième membre de la condition de stabilité.

Un premier moyen consiste évidemment à s'arranger pour donner au réseau un notable coefficient d'autoréglage, par exemple en donnant aux régulateurs de tension un statisme notable par rapport à la fréquence. C'est évidemment une solution, mais une solution qui tourne le problème sans le résoudre véritablement

Une solution véritablement satisfaisante serait celle qui «sans modifier la nature du réseau alimenté » permettrait de diminuer le deuxième membre de la condition de stabilité, donc d'augmenter la promptitude de réglage ou de diminuer l'inertie de l'alternateur sans diminuer la stabilité.

Je sais bien que les différents auteurs qui avaient étudié le problème de la stabilité des groupes hydroélectriques s'étaient trouvés tous d'accord pour admettre qu'il était "dans la nature des choses» qu'une grande stabilité soit payée par « une grande lenteur de réglage» et que tous les régulateurs de quelque type qu'ils soient, étaient logés à la même enseigne. Mais ces auteurs n'ont probablement pas voulu dire 
par là qu'il était impossible d'améliorer «la nature des choses» par un perfectionnement des régulateurs actuels.

\section{LE DÉTECTEUR MANOMÉTRIQUE}

\section{(Système DANIEL)}

Diminuer le coefficient $K$, tel était donc le problème auquel, suivant nos conseils, $M$. DANIEL, Ingénieur des Arts et Métiers, avait décidé de consacrer sa thèse de doctorat.

11 présenta ainsi son système en soutenant sa thèse en juillet 1947 devant l'Université de Grenoble.

L'idée originale de M. DANIEL était de faire agir à côté du tachymètre et de l'accéléromètre (ou du tachymètre et du dash-pot) un manomètre différentiel, détectant l'écart $\triangle h$ entre la pression actuelle et la pression moyenne. A cet effet, les deux prises de pression du manomètre différentiel sont l'une en communication directe avec lui : elle mesure la pression $H$ actuelle, l'autre en communication avec lui à travers un orifice étranglé et un réservoir d'air : elle mesure la pression moyenne $H_{0}$.

Considérons donc un régulateur comportant un tachymètre, un accéléromètre et un manomètre différentiel.

Sa loi de réponse sera :

1") $\frac{d}{d t} \Delta y=-\frac{1}{\tau} \Delta \omega-K_{1} \frac{d}{d t} \Delta \omega-K_{2} \Delta h$

On éliminera deux des variables $\Delta(),, \Delta y, \Delta h$ entre les trois équations $1^{\prime \prime}, 23$, et on trouvera l'équation différentielle:

$$
\begin{array}{r}
\frac{\tau y_{0} \theta}{2} \frac{d^{3}}{d t^{3}} \Delta x+\left(\tau-K_{1} y_{0} \theta-K_{2} \tau y_{0} \theta\right) \frac{d^{2}}{d t^{2}} \Delta x \\
+\left(K_{1}-\frac{y_{0} \theta}{\tau^{\prime}}\right) \frac{d}{d t} \Delta x+\frac{1}{\tau^{i}}=0
\end{array}
$$

L'application à cette équation des conditions de ROUTH ou d'HURWITZ conduit à la remarque qu'il suffit de choisir:

$$
\begin{aligned}
& K_{2} \tau>\frac{m}{\tau^{\prime}}=K_{1}>\frac{\theta}{K_{1}^{\prime}} \\
& K_{2}>\frac{-}{\tau}
\end{aligned}
$$

pour qu'on puisse réaliser la stabilité avec:

$$
z^{\prime}>\frac{0}{K_{1}}
$$

Comme rien n'empêche théoriquement de prendre $K_{2}$ et $K_{1}$ aussi grands qu'on le veut, on voit que :- pourra être aussi petit qu'on le veut.

II ne faudrait toutefois pas croire qu'on pourra ainsi rendre le réglage aussi rapide qu'on le voudra.

$$
\text { En effet, }- \text {, coefficient de } \Delta \text { a) dans la loi de }
$$
réponse I" n'est plus ici la véritable promptitude de réglage.

Si, en effet, on remplace dans (l") $\triangle \mathrm{h}$ par sa valeur donnée par (3), elle devient:

$$
\text { (1"') } \begin{aligned}
\frac{d}{d t} \Delta y=\frac{-\Delta \omega}{\tau^{\prime}\left(1+K_{2} \theta\right)} & -\frac{K_{1}}{1+K_{2} \theta} \frac{d}{d t} \Delta \omega \\
& -\frac{K_{2} \theta}{2\left(1+K_{2} \theta\right)} \frac{d}{d t} \Delta h
\end{aligned}
$$

La véritable promptitude de réglage - coefficient de $\triangle \omega$ - n'est pas $1 / \xi^{\prime}$ mais 1

$$
:^{\prime}\left(1+K_{2} 0\right)
$$

C'est donc la quantité :' $\left(1+K_{2} 0\right)$ qu'il y a lieu de rendre la plus petite possible si on veut accroitre les qualités de réglage du régulateur. La condition de stabilité s'écrit alors en appelant :

- : la véritable lenteur de réglage et en choisissant :

$$
\begin{aligned}
& K_{1} \text { et } K_{2} \text { très grands: } \\
& \frac{0}{2}>1
\end{aligned}
$$

Grâce à l'influence du détecteur manométrique DANIEL, la valeur du deuxième membre de la condition de stabilité (4) est passée de 3,74 à 1 , c'est-à-dire que sur une installation donnée ( : et $\theta$ donnés) le système DANIEL permettrait de multiplier par 3,74 la promptitude de réglage tout en gardant la même stabilité.

A promptitude égale, il permettrait de diviser par 3,74 le $\mathrm{Pd}^{2}$ (proportionnel à :) de l'alternateur sans nuire à la stabilité.

L'application du procédé DANIEL ne va pas sans difficultés. 
Nous avons vu, en effet, qu'il y avait intérêt à rendre $K_{1}$ et $K_{2}$ (coefficients d'actions accélérométrique et manométrique) les plus grands possible si l'on voulait lui donner l'efficacité maximum.

Par ailleurs, l'écart de pression $\triangle h$ est délicat à détecter surtout dans une Kaplan où l'écoulement de l'eau se fait avec une assez grande turbulence. Il serait nécessaire de construire un manomètre capable de ne retenir parmi les variations de pressions que celles dûes à l'inertie de l'eau et de rejeter celles dûes à la turbulence, ce qui est à la rigueur réalisable, par suite de leurs fréquences différentes. Cependant, il était intéressant de trouver autre chose, d'autant plus que l'efficacité du système manométrique prouvait bien que le problème n'était pas insoluble.

\section{LE DÉTECTEUR DE DÉRIVÉE SECONDE DE LA VITESSE}

En se rappelant les affirmations de nombreux spécialistes de la régulation - nous citerons en particulier MM. AILLERET et CAYERE suivant lesquelles la stabilité ne pouvait que gagner à la détection des dérivées successives du paramètre à régler, il était naturel de chercher à voir ce que pouvait donner un détecteur de dérivée seconde de la vitesse ajouté à un tachymètre et à un accéléromètre, dans une installation hydroélectrique où l'inertie de l'eau a une grande influence.

La loi de réponse du régulateur s'écrit alors:

$$
\left.1^{4}\right) \frac{d}{d t} \Delta y=\frac{1}{\tau^{2}} \Delta \omega-K_{1} \frac{d}{d t} \Delta \omega-K_{2} \frac{d^{2}}{d t^{2}} \Delta \omega
$$

ce qui, combiné avec les équations 2 et 3 donne l'équation différentielle:

$$
\begin{gathered}
\frac{y_{0} \theta \tau}{2}\left(1-2 \frac{K_{2}}{\tau}\right) \frac{d^{3}}{d t^{3}} \Delta x+\left(1+\frac{K_{q}}{\tau}-\frac{K_{1} y_{0}}{\tau}\right) \frac{d^{2}}{d t^{2}} \Delta x \\
+\left(K_{1}-\frac{\theta}{\tau^{\prime}}\right) \frac{d}{d t} \Delta x+\frac{1}{\tau^{\prime}}=0
\end{gathered}
$$

L'application à cette équation des conditions d'HURWITZ indique que la valeur minimum qu'on puisse donner à $\frac{\tau^{\prime}}{g^{2}}$ est $2 / 3$ ou : $\frac{\tau^{\prime}}{0^{\prime}}>\frac{2}{3}$ si l'on a eu soin de choisir conve-

nablement les facteurs $K_{1}$ et $K_{2.2}$ :

$$
\begin{aligned}
& \mathrm{K}_{2}=\mathrm{K}_{1} \frac{0}{3} \\
& \mathrm{~m}=\mathrm{K}_{1}:=0
\end{aligned}
$$

Le système DANIEL permettait de réduire la valeur de $\frac{\because}{g^{2}}$ de 3,74 à 1. L'introduction de la dérivée seconde parait encore plus favorable puisqu'elle permet de réduire cette quantité de 3,74 à $2 / 3$ donc de réduire le $P d^{\prime \prime}$ nécessaire à la stabilité dans le rapport 5,6 à 1 , sans diminuer la promptitude de réglage, ou encore, pour un $\mathrm{Pd}^{2}$ donné, de multiplier la promptitude de réglage $K_{0}=1 / \tau^{\prime} \operatorname{par} 5,6$

La différence entre les résultats obtenus par le détecteur manométrique d'une part, par le détecteur de dérivée seconde d'autre part, nous avait caché l'analogie profonde entre les deux systèmes.

En fait l'action du détecteur manométrique est identique à celle du détecteur de dérivée seconde; la différence entre les gains dus aux deux systèmes n'est qu'apparente : elle provient du manque de précision de la notion de promptitude ou lenteur de réglage. Cette notion s'est trouvée fort judicieusement précisée dans un article publié dans la Revue Cénérale d’Electricité (1) sous la signature de M. DEJOU, du Service "Recherches» de l'Electricité de France, avec qui nous avons eu d'ailleurs le plaisir de collaborer plusieurs semaines à des recherches de ce genre lors d'un stage qu'il accomplit chez Neyrpic en octobre 1947.

On a donc là un moyen théorique d'améliorer les réactions des régulateurs des groupes hydroélectriques devant les phénomènes dus à l'inertie de l'eau. Nul doute qu'on puisse trouver des moyens de détection de la dérivée seconde de la vitesse par des moyens mécaniques, hydrauliques ou électriques, mais cela posait le problème de la construction d'un appareil nouveau.

(1) On trouvera aussi dans cet article une étude détaillée des comportements de régulateurs munis soit d'un détecteur de dérivée seconde, soit d'un tachyaccéléromètre à dash-pot. (Août 1948 , 
LE

\section{RÉCULATEUR TACHYACCÉLÉROMÉTRIQUE A ASSERVISSEMENT TEMPORAIRE}

Cependant, nous basant sur le fait que le régulateur à asservissement temporaire était, à condition quion lui donne des caractéristiques convenables, équivalent au régulateur accélérotachymétrique, nous avons été tout naturellement conduits au régulateur mixte accélérotachymétrique avec asservissement temporaire.

« Ce nouveau système n'est pas un régulateur accélérotachymétrique auquel on a ajouté un asservissement temporaire, mais un régulateur à asservissement temporaire dans lequel on a remplacé le tachymètre par un système mixte détectant vitesse et accélération ".

La différence entre les deux réside dans la conception même de leur tiroir de distribution. différence qui est fondamentale (voir HOUILLE BLANCHE No 6/1947).

Par une mise en équation analogue à celle que nous avons faite pour le régulateur à asservissement temporaire, on trouve une loi de réponse:

$$
\frac{\left.1^{5}\right)}{d t} \Delta y=-\frac{1}{\sigma T_{r}} \Delta \omega-\left(\frac{m+T_{r}}{\sigma T_{r}}\right) \frac{d}{d t} \Delta \omega-\frac{m}{\sigma} \frac{d^{2}}{d t^{2}} \Delta \omega
$$

II suffit donc de remplacer dans l'équation différentielle relative au régulateur à détecteur de dérivée seconde, :'par $T_{r} \sigma, m=K_{1}$ : $^{\prime}$ par $m+T_{r}$ ou $K_{1}$ par $\frac{m+T_{r}}{T_{r} \tau}$ et $K_{2}$ par $\frac{m}{\sigma}$ d'où l'équation:

$$
\begin{aligned}
\frac{y_{0} \theta \tau}{2}\left(1-2 \frac{m}{\sigma}\right) \frac{d^{3}}{d t^{3}} \Delta x & +\tau\left[1+\frac{m}{\sigma}-\frac{\left(m+T_{r}\right) y_{0} \theta}{T_{r}}\right] \frac{d^{2}}{d t^{2}} \Delta x \\
& +\frac{m+T_{r}-y_{0} \theta}{T_{r} \sigma} \frac{d}{d t} \Delta x+\frac{1}{T_{r} \sigma}=0
\end{aligned}
$$

Etant donné l'identité des équations, on pourrait croire que le gain réalisé avec ce procédé est le même qu'avec le précédent. Mais, en réalité, les conditions optima du cas précédent :

$$
\begin{aligned}
& m=0 \\
& \frac{K_{2}}{K_{1}}=\frac{0}{3}
\end{aligned}
$$

s'écrivent ici :

$$
\begin{aligned}
& m+T_{r}=g \\
& m T_{r}=\frac{}{3}
\end{aligned}
$$

système qui ne comporte aucune racine réelle en $m$ et $T$.

On en est alors réduit, pour s'approcher le mieux possible des conditions optima, à réaliser la condition:

$$
\begin{aligned}
& m=T_{r}=\frac{20}{3} \\
& \sigma<4 / 3 \frac{0}{-} \\
& \div \\
& \text { ou : : }=z \mathrm{~T}_{\mathrm{r}}>\frac{8}{9}= \\
& \text { ou }: \frac{y^{\prime}}{g^{2}}>\frac{8}{9}
\end{aligned}
$$

Ce système permet donc de réduire dans une installation la valeur de $\frac{\because{ }^{2}}{g^{2}}$ dans le rapport de 3,74 à $8 / 9$ (soit de 4,2 à 1 ).

II n'est donc théoriquement pas aussi efficace que le précédent, mais il est beaucoup plus simple à construire.

Ce qui vient d'être dit met bien en évidence qu'il s'agit bien, au fond, d'un régulateur à asservissement temporaire, auquel on a ajouté un accéléromètre : sa promptitude de réglage est la même que celle du régulateur à asservissement primitif.

\section{CONCLUSION}

On dispose donc de trois moyens théoriques de multiplier la promptitude de réglage d'un groupe hydroélectrique - ou de diviser le $\mathrm{Pd}^{2} \mathrm{du}$ groupe - par 3,$74 ; 5,6$; et 4,2 sans diminuer sa stabilité.

On peut hésiter entre ces trois systèmes (1), quoique pour le moment il semble que ce soit le dernier régulateur à asservissement temporaire avec détecteur accélérotachymétrique qui semble le plus facile à réaliser, puisqu'il ne comporte pas d'appareils nouveaux, mais seulement une combinaison nouvelle d'appareils connus.

On peut se demander, surtout pour les deux derniers systèmes, comment il se fait qu'on n'ait

(1) Les procédés d'amélioration du réglage des groupes hydroélectriques dont il est question dans cet article, ont fait l'abjet de differents brevets pris conjointement par les Etablissements NEYRPIC et $M$. DANIEL. 
pas songé à le réaliser plus tôt. La réponse est facile. Jusqu'à ces derniers temps, le problème ne se posait pas. On s'était bien apercu que certaines installations ne pouvaient être stables qu'au prix d'une diminution de leurs qualités de réglage, mais ces groupes étaient malgré tout en minorité de sorte que dans l'ensemble du réseau interconnecté, la fréquence était suffisamment bien réglée. Le problème n'a commencé à se poser avec acuité que le jour où on a construit de nombreuses centrales de basses chutes et de grande puissance et que ces centrales ont vu leur puissance grandir par rapport à la puissance totale du réseau et aussi lors de l'équipement hydroélectrique de pays neufs. Notamment, le problème serait particulièrement aigu si l'on se mettait à construire les installations marémotrices actuellement envisagées.
Ceci est d'autant plus vrai qu'au même moment les progrès dans la technique de construction électrique permettent aux électriciens d'alléger leurs alternateurs, donc de diminuer leur Pd" « économique ».

Les constructeurs hydrauliciens ont été déjà conduits, pour des installations de basse chute à axe vertical où il n'est pas question d'admettre un volant, à demander aux constructeurs électriciens des $\mathrm{Pd}$ " disproportionnés avec les $\mathrm{Pd}$ " «éco. nomiques $\gg$.

C'est ainsi qu'il est courant que le Pd' réclamé par l'hydraulicien soit le double ou le triple du Pd" économique.

Les systèmes décrits ci-dessus permettraient donc de retrouver une bonne harmonie entre les désiderata des électriciens et des hydrauliciens.

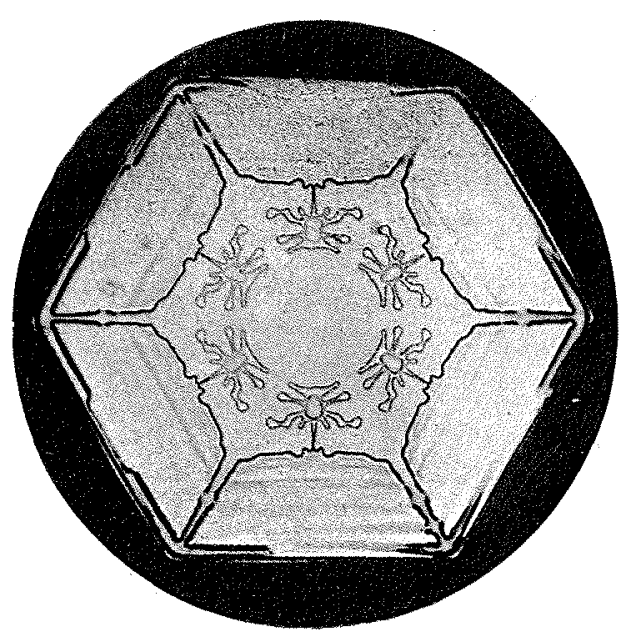

